\title{
Ballistic current induced effective force on magnetic domain wall
}

\section{Wang and K. Xia*}

The collective dynamics of magnetic domain wall under electric current is studied in the form of spin transfer torque (STT). The out-of-plane STT induced effective force is obtained based on the Landau-Lifshitz-Gilbert (LLG) equation including microscopic STT terms. The relation between microscopic calculations and collective description of the domain wall motion is established. With our numerical calculations based on tight binding free electron model, we find that the non adiabatic out-of-plane torque components have considerable non-local properties. It turns out that the calculated effective forces decay significantly with increasing domain wall widths.

Keywords: Magnetic domain; Spin Transfer torque; Spintronics; First principle study

Citation: C. Wang and K. Xia, "Ballistic current induced effective force on magnetic domain wall”, Nano-Micro Lett. 1, 34-39 (2009). doi: 10.5101/nml.v1i1.p34-39

The application of spintronics [1] requires effective ways of spin manipulations in nanoscale devices. Recently, growing interest has been focused on the current induced magnetic dynamics. Electric currents can be spin-polarized by passing through magnetic domains, and the polarized spins of the conducting electrons can exert spin transfer torque (STT) to the localized magnetizations when going through a magnetic domain wall. On the other hand, the spins of electrons will be altered by the localized magnetizations in return [2,3]. Beautiful experiments have been conducted using STT effect in devices [4-7]. However, the dynamics of magnetic domain walls (DWs) in the presence of electric current are still not well understood, especially for the components of spin transfer torques perpendicular to the plane where the local magnetization's directions change. Shibata et al [8,9], studied the DW motion based on the s-d electron model and collective coordinates of the DW. They found that the DW motion will be dominated by the effective force on the DW, which is rooted from the reflection of the electrons incident on the DW. On the other hand, S. Zhang and Z. Li $[10,11]$ pointed out that the out-of-plane STT was crucial for the stead current induced DW motion and proposed a non-adiabatic STT due to spinrelaxation mechanism. Another non-adiabatic STT was proposed by J. Xiao [12], which is due to the misalignment of non-equilibrium spin density and local effective field. The out-plane spin transfer torques can stem from either spin relaxation or non-local non-adiabatic effects [9]. However, the link between the microscopic STT and the effective force on the DW is missing.

In this paper, we start from the Landau-Lifshitz-Gilbert (LLG) equation, including the microscopic STT Terms from our numerical calculation, theoretically analyze the motion of the DW. The relation between the effective forces on DW and STT are established. The connections between the different components of STT and the effective forces and STTs on domain wall are established. Then we discuss the relationships between our results and other models in the end of Section II. In Section III the STT in DW is studied numerically for free electron model. Our calculations of out-plane torques show that the parameter $c_{j}$ describing the non-adiabatic torque in LLG equation is not constant but position dependent, implying its non-local characters. The DW width dependence of the 
effective force is obtained.

\section{EFFECTIVE FORCE AND OUT-OF-PLANE STT}

The current-driven domain wall dynamics is studied based on the Landau-Lifshitz-Gilbert equation, the connection between effective force and out-of-plane STT on each site is obtained. The LLG equation reads,

$\frac{\partial}{\partial t} \vec{M}=-\gamma \vec{M} \times \vec{H}_{e f f}+\frac{\alpha}{M_{S}} \vec{M} \times \frac{\partial}{\partial t} \vec{M}+\bar{T}_{\|}+\bar{T}_{\perp}$

where the magnetization $\boldsymbol{M}$ can be written as the function of polar angles as indicated in Fig. 1:

$$
\vec{M}(\theta, \varphi)=M_{S}(\sin \theta \cos \varphi, \sin \theta \sin \varphi, \cos \theta)
$$

We assume that the magnitude of the magnetization will

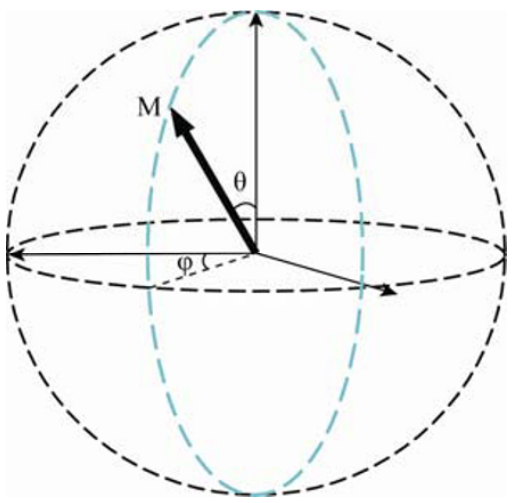

FIG. 1. Sphere polar coordinates describing the direction of localizec magnetization. M represent on-site local magnetization, the blue circle illustrate the plane in which magnetizations alter their directions. In the simplest model $(\varphi=0)$, the plane reduces to $\mathrm{x}-\mathrm{z}$ plane.

not change when the DW moves. We can rewrite the on sites LLG equations Eq. (1) with sphere polar angle coordinates $\left(\theta_{i}\right.$, $\left.\varphi_{i}\right)$ and then apply the Walker ansatz so that Euler angles are treated as functions of position and time.

The DW is parallel to the $z$ direction, $\theta$ and $\varphi$ are the function of position and time. $\theta=\theta(z-X(t)), \varphi=\varphi(z, t), \quad X$ is defined as the wall center's position within the "rigid wall" approximation, and $\varphi(z, t)$ is simply reduced to $\varphi(t)$ in the model used in following derivation. The variable $\mathrm{X}$ and $\varphi$ can serve as collective coordinates to describe the wall motion. Namely, we can get the dynamic information of the domain wall if we know the time evolution of $(X(t), \varphi(t))$. (In the simplest picture, we may let $\varphi$ constant so that the localized spins change their orientations within a plain). The effective field on each local magnetization

$$
\begin{aligned}
\vec{H}_{e f f, i} & =\vec{H}_{e x, i}+\vec{H}_{K, i}+\vec{H}_{\text {demag }, i}+\vec{H}_{e x t} \\
& =H_{e x} 4 \pi M_{S}\left(\vec{m}_{i-1}+\vec{m}_{i+1}\right)+H_{K} m_{z, i} \vec{e}_{z}-4 \pi M_{S} m_{x, i} \vec{e}_{x}+\vec{H}_{e x t}
\end{aligned}
$$

contains respective contributions from exchange interaction, anisotropy, demagnetization field, and external field where subscript $i$ denotes different sites in the wall. The nearest neighboring approximation and assumption of constant magnitude of $\boldsymbol{M}$ were applied. The unit vectors in Cartesian coordination can be transformed into polar coordination. And spin transfer torques can be explicitly expressed as,

$$
\overrightarrow{T_{\|}}=-b_{j} \frac{1}{M_{S}^{2}} \vec{M} \times\left(\vec{M} \times \frac{\partial \vec{M}}{\partial z}\right), \overrightarrow{T_{\perp}}=-c_{j} \frac{1}{M_{S}} \vec{M} \times \frac{\partial \vec{M}}{\partial z},
$$

Putting all the above terms into the Eq. (1), we eventually obtain the LLG equation written in polar coordination with variable $(\theta, \varphi)$ as,

$$
\begin{gathered}
\overrightarrow{e_{\theta}}: \dot{\theta}=\gamma 2 \pi M_{S} \sin \theta \sin 2 \varphi-\alpha \varphi \sin \theta \\
+T_{\| x} / M_{S} \cos \theta-T_{\| z} \sin \theta / M_{S} \\
\overrightarrow{e_{\varphi}}: \dot{\varphi} \sin \theta=\gamma\left(H_{e x} M_{S} 2 \pi \frac{-1}{\lambda^{2}} a^{2}+H_{K} / 2+2 \pi M_{S} \cos ^{2} \varphi\right) \\
\cdot 2 \sin \theta \cos \theta+\alpha \dot{\theta}+T_{\perp} / M_{S}
\end{gathered}
$$
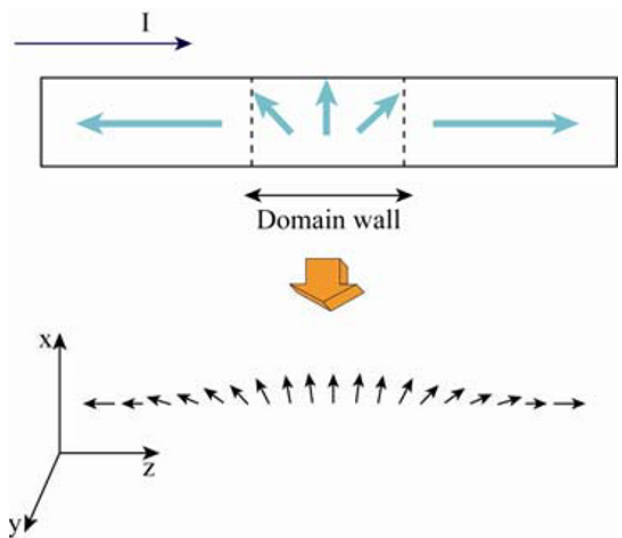

FIG. 2. Illustration of Neel domain wall structure and Cartesiar coordinates. The direction of current I in CIP structure used in ou calculation is also shown.

The above two sets of equations can be solved numerically for arbitrary STT form and effective field. However, when current and applied magnetic fields are not very large, rigid wall is a good approximation to study the DW motion. Applying Walker's ansatz [16], the localized spins in Neel domain wall structure (Shown in Fig. 2) expressed in spin polar coordination satisfies

$$
\cos \theta_{s}(z, t)=\tanh \left(\frac{z-X(t)}{\lambda}\right)
$$

Considering the magnetization $\vec{M}$ of the domain wall and 
the localized macroscopic spin $\vec{S}$ satisfy the relationship: $\vec{M}=-\gamma \vec{S} / a^{3}$, we can express this relationship by pole angles: $\cos \left(\pi-\theta_{s}(z, t)\right)=\tanh \left(\frac{z-X(t)}{\lambda}\right), \varphi_{M}(z, t)=\varphi_{M}(t)$. And we can see the time dependent behavior of $\theta$ is completely determined by the wall(center) position's dynamics property:

$$
\dot{\theta}_{M}=-\dot{\theta}_{S}=-\frac{1}{\lambda} \sin \theta_{s} \dot{X}
$$

For the convenience in connecting to our first principles calculation, we use the $\vec{S}$ in the following of the paper. Using the relationship of magnetic moment and spin angular momentum $M_{S}=\gamma_{0} \frac{1}{a^{3}} S$, the Eq. (5) (6) could be expressed by localized macroscopic spins. Summing all the equations on each site together, we obtained the corresponding equations for the collective coordinates. Replacing the summation by integration for the simplicity, the region of integration is set as where $\mathrm{L}=\mathrm{Nd}$ is the wall length, $d$ is the distance between neighboring sites, and $N$ is the total number of sites in a domain wall which can be extended to $\infty$. Since $\cos \theta$ varies from -1 to 1 in a symmetric way, the sum over position is $\int \cos \theta(z, t) d z=0$. Considering the two collective coordinations $(X, \varphi)$ are independent on position, the sum of equations on all the sites along the wall results in

$$
\begin{aligned}
\overrightarrow{e_{\theta}}: \dot{X}-\alpha \lambda \dot{\varphi}= & 2 \pi \frac{\gamma \gamma_{0}}{a^{3}} S \lambda \sin 2 \varphi \\
& +\frac{\lambda}{S L} \int_{-L / 2}^{L / 2} d z\left(\frac{\cos \theta}{\sin \theta} T_{\| x}-T_{\| z}\right) \\
\overrightarrow{e_{\varphi}}: \dot{\varphi}+\alpha \frac{1}{\lambda} \dot{X}= & \frac{1}{S L} \int_{-L / 2}^{L / 2} d z \frac{1}{\sin \theta} T_{\perp}
\end{aligned}
$$

So the equations portraying the dynamic behavior of the rigid wall are obtained. Recalling the expressions of spin torque in LLG Eq. (4), the spin torque exerted on localized spins in the right side of equations above can be simplified by

$$
\left(T_{\| x} \frac{\cos \theta}{\sin \theta}-T_{\| z}\right) / S=\frac{1}{\lambda} b_{j}, T_{\perp} \frac{1}{S \sin \theta}=\frac{1}{\lambda} c_{j}
$$

With the obtained results, we can immediately obtain the velocity, effective force and torques exerted on the domain wall in the following. If we regard $b_{j}$ and $c_{j}$ are independent on position (we will discuss the validity of this simplification with our calculations section III), the current-driven domain wall motion velocity, as the time derivative of wall position $X$, can be expressed by $b_{j}$ and $c_{j}$ using Eq. (8) (9) (10):

$$
\dot{X}=-\frac{1}{\alpha^{2}+1}\left(b_{j}+\alpha c_{j}\right)
$$

Our results Eq. (9) and (10) also imply that for $t \rightarrow \infty$ in which $\dot{\varphi}=0$, the magnitude of the domain wall ultimate velocity is determined by $c_{j}$ while the initial speed is mainly determined by $b_{j}$. The expression of velocities agrees well with other groups' previous outcome [10]. In order to further clarify the roles of different components of STT, we combine our results with what obtained from s-d model Hamiltonian $H_{s d}=-J_{s d} \int d^{3} \mathbf{x} \mathrm{S} \cdot \mathbf{s}$. We can immediately come to some interesting results after comparing Eq. (8) (9) with effective forces and effective torques [9]. It turns out that the out-plane spin torque $T_{\perp y}$, which appears in Eq. (9) corresponds to the force on domain wall, while the in-plane spin torque $T_{\|}$contributes to the $z$ component of torque on the wall as a whole:

$$
\begin{aligned}
& \frac{\lambda}{N_{w} S} \tau_{z}=\frac{\lambda}{S L} \int_{-L / 2}^{L / 2} d z\left(\frac{\cos \theta}{\sin \theta} T_{\| x}-T_{\| z}\right) \\
& \frac{\lambda}{N_{W} S} F=\frac{d}{\lambda S L} \int_{-L / 2}^{L / 2} d z \frac{1}{\sin \theta} T_{\perp}
\end{aligned}
$$

Eq. (12) tells that force exerted on the rigid domain wall is also originated from the STT(but only the perpendicular element $T_{\perp y}$ ), while Eq. (13) shows that the summed effective spin transfer torque along $\mathrm{z}$ is deter-mined by integration involving different positions' $T_{\| x}$ and $T_{\| z}$. After we apply Eq. (10) and define $\overline{b_{j}}$ and $\overline{c_{j}}$ as the average values of the two parameters, the two integrations in Eq. (12) and Eq. (13) become

$$
\begin{gathered}
\frac{\lambda}{N_{w} S} \tau_{z}=\overline{b_{j}} \\
\frac{\lambda}{N_{w} S} F=\frac{1}{\lambda} \overline{c_{j}}
\end{gathered}
$$

Although it is widely accepted that different components of STTs can be written in the form of Eq. (4), the origin of out-of-plane STT could be stem from different physics. In our calculation, we focused on the nonadiabatic term pointed out by J. Xiao and M. Stiles [12].

\section{NUMERICAL CALCULATION AND DISCUSSION}

In the following, numerical calculation is used to 
investigate the torque as the function of domain wall width $\lambda$. After introducing the calculation methods we used, we subsequently investigate the in-plane and out-plane STTs. The obtained calculations are in accordance with our model and can be helpful to the discussion about effective force.

\section{A. Calculation of STT in Domain Wall}

The structure used in the calculation is current in plane (CIP) domain walls in ferromagnetic materials. We apply free electron model in our calculations where the current is along (110) direction in fcc structure using the lattice constant of Cobalt $\mathrm{a}_{\mathrm{Co}}=3.549 \AA$. And we set the energy split between majority and minority spins in free electron energy band as $1.69 \mathrm{eV}$, which equals the value of exchange energy split of bulk Cobalt. Our numerical approach is based on the Tight-binding linear muffin-tin orbital formulism [13,14]. Scattering wave function is obtained by the wave function matching method [15]. The rigid potential approximation is employed to simulate the DW. Here STTs in our study can be defined as the difference between the incoming and outgoing spin current at $\mathrm{R}$ site.

$$
\overrightarrow{T_{R}}=\left(\frac{\hbar}{2}\right) \frac{e}{\hbar} \frac{1}{N_{\|}} \sum_{s, k_{\|}}\left\langle\widehat{T}_{R}^{s}\left(k_{\|}\right)\right\rangle V_{b}
$$

Here summation over the 2D Brillouin zone is performed. Combining with the expression of current passing through R-site, the STT per unit current is obtained:

$$
\vec{T}=\frac{\overrightarrow{T_{R}}}{G \cdot V_{b}}
$$

Given a specific current density $\left(\sim 10^{10} \mathrm{~A} / \mathrm{m}^{2}\right)$, we can calculate corresponding torque. The calculated spin transfer torques along the DW is presented in different components in Fig. 3. From the Eq. (12) and Eq. (13) the force and

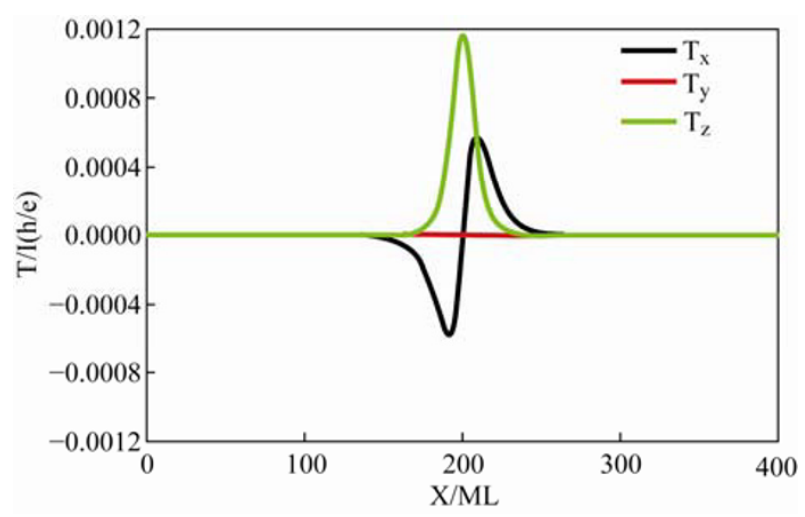

FIG. 3. Position dependence of different components of spin transfer torque in the domain wall structure. z-component's effective torque can be calculated.

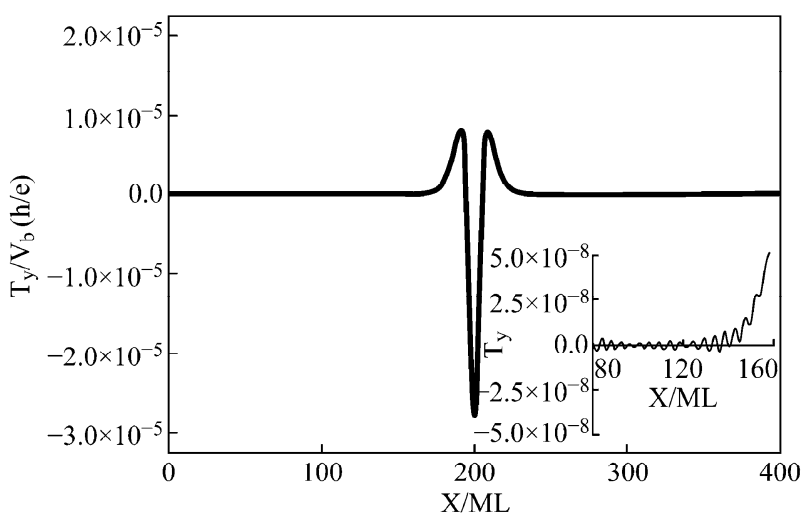

FIG. 4. Distribution of out-plane STT components in DW with $\lambda=6 \mathrm{ML}$. The inset shows the decaying oscillations of Ty at sites away from DW centers.

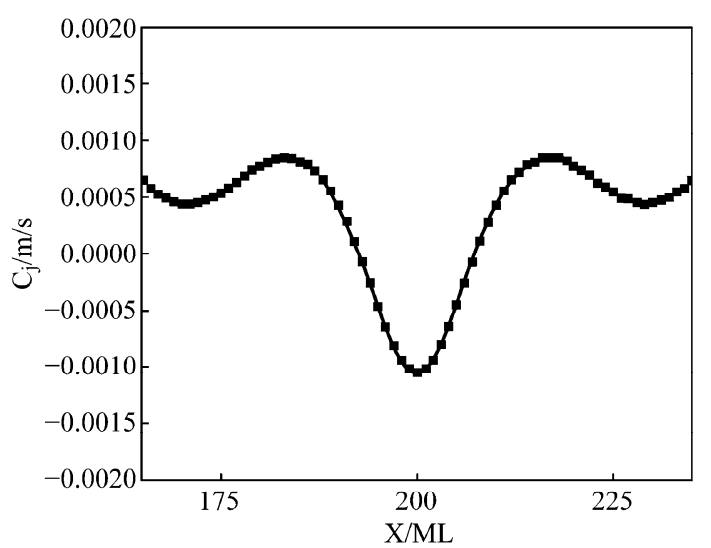

FIG. 5. The values of $c_{j}$ near the wall center. We make a cutoff and only consider the contribution of sites not far from the wall center.

\section{B. Out-plane STT and Effective Force}

Let us focus on the out-plane component of STTs. From our numerical calculations, we find a decaying oscillation for out-of-plane STT $T_{\perp y}$ as shown in Fig. 4. From the inset in Fig. 4 we can see the decaying oscillation of $T_{\perp y}$ at the sites away from DW centers. We can see that $T_{\perp y}$ deviate significantly from zero only within a certain distance from wall center. We can fit these STTs with Eq. (10) to obtain the on site parameter $c_{j}=\lambda T_{\perp y} /(\mathrm{S} \sin \theta)$. Numerical difficulty comes from the position far away from the DW center, where $\sin \theta(z, t)$ is very small and out-of-plane STTs oscillate as the function of position. Considering that the STT mainly dominated by the DW near the center where the angle of local magnetization changes significantly, a cut off is applied so that our focus is 


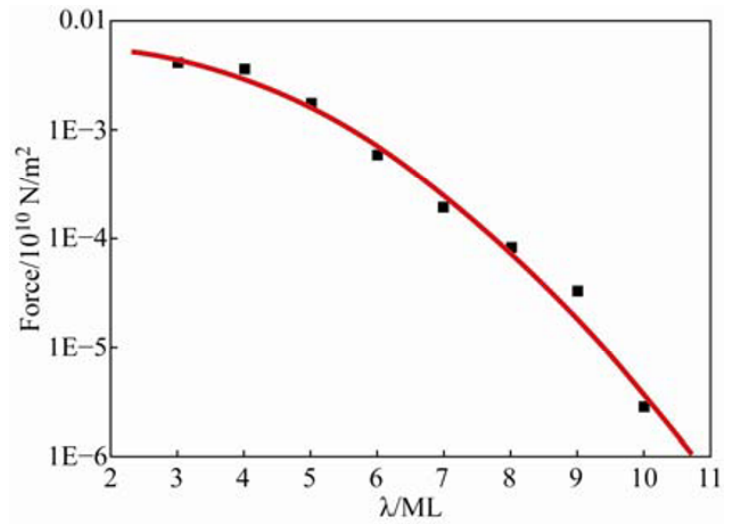

FIG. 6. The force is calculated in wall structures with different widths. Our average $c_{j}$ only take account of the contributions from sites near the wall center where the value of $\sin \theta(z, t)$ is nonzero. The red line is a parabolic exponential curve.

concentrated to regions near the wall centers. The calculated $c_{j}$ as a function of position is presented in Fig. 5. From Fig. 5 we can see that $c_{j}$ obtained from our model are position dependent rather than constant. This is reasonable because the non-adiabatic torques is nonlocal and has vibrating behaviors. Combining the calculated results, we will discuss the DW velocities expressed in Eq. (11). When the rigid wall still holds, it is suitable to express the terminal velocities $v(t \rightarrow \infty)=-c_{j} / \alpha$.

Considering $c_{j}$ is not constant but oscillating, we may use the average $\overline{c_{j}}$ to express the terminal velocity. When the wall is thicker, the increase of inertia or wall mass makes it harder to move, thus the velocities get smaller and consequently larger driving currents are required. In our model, the velocities will indeed drop significantly when the out plane torques ( $c_{j}$ terms) vanish in thicker walls, as depicted in Fig. 6. However, we need to keep in mind that the Walker's model we use to describe rigid walls will break down under high external field [16,17] or high current density [9]. In that case, the domain wall would be oscillating so the definition of DW velocities is no longer available.

The forces at different wall structures are calculated from integration within the narrowed region of $c_{j}(\mathrm{z})$ using $F=\left(S / \lambda^{2}\right) \sum_{n} c_{j}\left(z_{n}\right)$ and presented in Fig. 6. We can see from the result that the forces exerted on the walls decrease significantly as the walls get thicker, implying that the non-adiabatic effect is obvious in thin domain walls. And the forces or the integrated non-adiabatic torques decay approximately parabolic exponentially with the increase of DW width.

\section{CONCLUSION}

In sum, domain wall motion under electrical current is studied focusing on spin transfer torque effect. Under the approximation of rigid wall the relationships between the force, velocity and different components of STT are clarified. And the results of our calculation successfully testify the validity of adiabatic limit. For out-plane component, the calculated torques show oscillating and decaying behaviors. The calculated non-adiabatic torque dependence on wall width is in good accordance with the physical illustration that an extremely thick wall would become completely adiabatic and thus the forces and terminal velocities origined from non-adiabatic terms vanish.

The work is supported by NSF of China (Grant No.60825405) and MOST of China (2006CB933000). We are grateful to Shuai Wang for useful discussions and his codes that we used for calculating spin transfer torques.

Received 24 September 2009; accepted 5 December 2009; published online 20 December 2009.

\section{References}

1. S. A. Wolf, D. D. Awschalom, R.A. Buhrman, J. M. Daughton and S. Von Molnar, Science 294, 1488 (2001); I. Zutic, J. Fabian and S. Das Sarma, Rev. Mod. Phys. 76, 2 (2004).

2. Berger, Phys. Rev. B 54, 9353 (1996). doi:10.1103/ PhysRevB.54.9353

3. J. Slonczewski and J. Magn. Magn. Mater. 159, L1 (1996). doi:10.1016/0304-8853(96)00062-5

4. G. S. D. Beach, C. Knutson, C. Nistor, M. Tsoi and J. L. Erskine, Phys. Rev. Lett. 97, 057203 (2006). doi:10.1103/PhysRevLett.97.057203

5. M. Tsoi, Nature Phys. 4, 17 (2008). doi:10.1038/nphys $\underline{822}$

6. J. C. Sankey, P. M. Braganca, A. G. F. Garcia, I. N. Krivorotov, R. A. Buhrman and D. C. Ralph, Phys. Rev. Lett. 96, 227601 (2006). doi:10.1103/PhysRevLett. $\underline{96.227601}$

7. S. Stuart and P. Parkin, Science 320, 190 (2008). doi:10.1126/science.1145799

8. J. Shibata, G. Tarara and H. Kohno, Phys. Rev. Lett. 94, 076601 (2005). doi:10.1103/PhysRevLett.94.076601

9. G. Tatara, H. Kohno and J. Shibata, Phys. Rep. 468, 6 (2008). doi:10.1016/j.physrep.2008.07.003 
10. Z. Li and S. Zhang, Phys. Rev. Lett. 93, 127204 (2004). doi:10.1103/PhysRevLett.93.127204

11. S. Zhang and Z. Li, Phys. Rev. Lett 92, 207203 (2004). doi:10.1103/PhysRevLett.92.207203

12. J. Xiao, A. Zangwill and M. D. Stiles, Phys. Rev. B. 73, 054428 (2006). doi:10.1103/PhysRevB.73.054428

13. I. Turek, V. Drchal, J. Kudrnovsky, M. Sob and P. Weinberger, Electronic Structure of Disordered Alloys: Surface sand Interfaces (Kluwer, Boston-LondonDordrecht, 1997).
14. O. K.Andersen, O. Jepsen and D. Glotzel, in Highlights in Condensed Matter Theory, edited by F. Bassani, F. Fumi and M. P. Tosi (North-Holland, Amsterdam, 1985), p. 59.

15. S. Wang, Y. Xu and K. Xia, Phys. Rev. B 77, 184430 (2008). doi:10.1103/PhysRevB.77.184430

16. N. L. Schryer and L. R. Walker, J. Appl. Phys. 45, 5406 (1974). doi:10.1063/1.1663252

17. X. R. Wang, P. Yan, J. Lu and C. He, Ann. Phys. 324, 8 (2009). doi:10.1016/j.aop.2009.05.004 